\title{
Search for a stable six-quark state in $\Upsilon$ decays at $B A B A R$
}

\author{
Gerald Eigen * \\ University of Bergen \\ E-mail: gerald.eigen@uib.no
}

Recent investigations have suggested that the six-quark combination uuddss could be a deeply bound state $(S)$ that has eluded detection so far, and a potential dark matter candidate. We report the first search for a stable, doubly strange six-quark state in $\Upsilon \rightarrow S \bar{\Lambda} \bar{\Lambda}$ decays with the BABAR experiment. No signal is observed, and limits on the combined $\Upsilon(2 S, 3 S) \rightarrow S \bar{\Lambda} \bar{\Lambda}$ branching fraction set stringent limits on the existence of such exotic particles.

European Physical Society Conference on High Energy Physics - EPS-HEP2019 -

10-17 July, 2019

Ghent, Belgium

* Speaker.

$\dagger$ On behalf of the BABAR Collaboration. This work was supported by Norwegian Research Council. I would like to thank the BABAR collaboration for the opportunity to give this talk, in particular Janis McKenna, Racha Chealb and Bertrand Echenard for useful comments on the slides and Frank Porter for checking the proceedings. 


\section{Introduction}

In the traditional quark model, only mesons $(q \bar{q})$ and baryons $(q q q)$ are predicted [1]. Other states such as $(q \bar{q} q \bar{q}),(q \bar{q} q q)$, or $(q q q q q q)$ were not considered since those were not observed. In recent years, however, several experiments found states consistent with tetraquarks $(q \bar{q} q \bar{q})$. For example, the Belle experiment first observed the $X(3872)$, a $J^{P C}=1^{++}$state in $B \rightarrow K \pi^{+} \pi^{-} J / \psi(1 S)$ [2]. Figure 1 (left) shows the $J / \psi \pi^{+} \pi^{-}$invariant-mass spectrum, which reveals a prominent peak at $3872 \mathrm{MeV} / \mathrm{c}^{2}$. The state has exotic properties that differ from a conventional $q \bar{q}$ state. The classification as a tetraquark state is the most likely option. The BES III experiment first observed the $Z_{c}(3900)^{+}$state [3] and Belle first observed the $Z_{c}(4430)^{+}$state, which have properties that are incompatible with a $q \bar{q}$ assignment. These states are consistent with being tetraquark states as well. By now, the LHCb experiment observed three pentaquark states, the $P_{c}(4312)$, the $P_{c}(4440)$ and the $P_{c}(4457)$ [5]. They are visible in the $J / \psi p$ invariant-mass spectrum displayed in Fig. 1 (right). Therefore, it is rather likely that also six-quark states may exist.
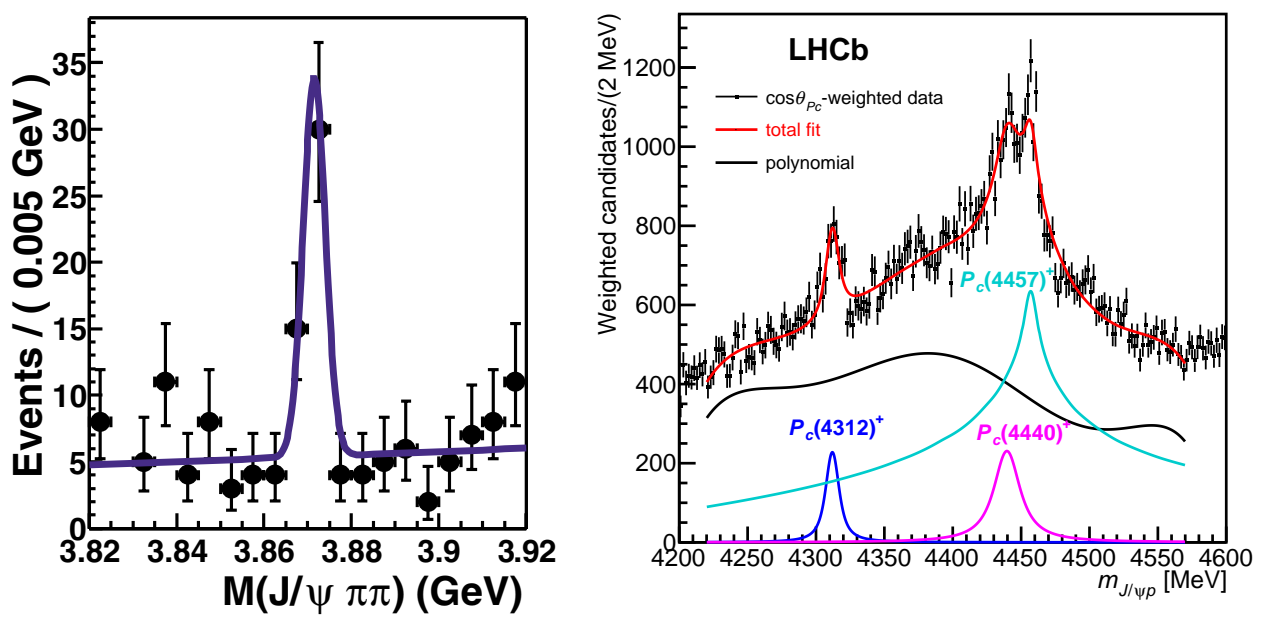

Figure 1: Left: The $J / \psi \pi^{+} \pi^{-}$invariant-mass spectrum observed by Belle showing the $X(3872)$ exotic state. Right: The $J / \psi p$ invariant-mass spectrum from LHCb showing three pentaquark states, the $P_{c}(4412)^{+}, P_{c}(4440)$ and $P_{c}(4457)$ states [5].

In 1977, Jaffe proposed the H-dibaryon, a $|u d s u d s\rangle$, S-wave and flavor singlet state that is a loosely bound $\Lambda \Lambda$ state [7]. The bag model predicted its mass to be $m_{H}=2150 \mathrm{MeV} / \mathrm{c}^{2}$. If $m_{H}<2 m_{\Lambda}$ the state is stable with respect to strong decays. Therefore, the $\mathrm{H}$ is expected to decay weakly, with a lifetime of $10^{-10} \mathrm{~s}$. Numerous searches for an H-dibaryon, however, did not find any signal.

In 2017, Farrar proposed a $|u u d d s s\rangle$ six-quark state $\mathrm{S}$ that is different from the loosely bound $\mathrm{H}$-dibaryon state at $2150 \mathrm{MeV}$ predicted by Jaffe. The scalar state has charge zero, baryon number two, strangeness minus two, and should be produced in decays such as $\Upsilon \rightarrow$ gluons $\rightarrow S \bar{\Lambda} \bar{\Lambda}$ [9]. It is a very compact state with a radius of $0.1-0.4 \mathrm{fm}$. Recent Lattice QCD calculations indicate that it is a tightly bound $\Lambda \Lambda$ state with a mass $m_{S}<m_{\Lambda}+m_{p}+m_{e}=2.0545 \mathrm{GeV} / \mathrm{c}^{2}$. if $m_{S}<$ $1.878 \mathrm{GeV} / \mathrm{c}^{2}, S$ is absolutely stable. It is a flavor singlet that is allowed by QCD with small couplings to mesons. Figure 2 shows the binding energy of various di-baryon systems calculated in lattice gauge theories [10]. Note that the singlet state is the most stable di-baryon state. The 
$S$-nucleon interactions are suppressed due to the tiny wave function overlap. Thus, neutron stars do not decay to $S$. Also, the decay $n n \rightarrow S \pi^{0}$ is not observed, Searches of past experiments rule out $m_{S}>2 \mathrm{GeV} / \mathrm{c}^{2}$. However, the mass region below $2 \mathrm{GeV} / \mathrm{c}^{2}$ have not been explored.

The six-quark $S$ state could be the astronomical dark matter (DM). If dark matter consists of a nearly equal number of $u, d, s$ quarks, the formation rate is driven by a quark-gluon plasma transition to the hadronic phase. So DM models in this category include hexaquarks. Please note that hexaquark DM with a mass of $1860-1880 \mathrm{MeV} / \mathrm{c}^{2}$ can reproduce the ratio of DM to ordinary matter densities $\Omega_{D M} / \Omega_{b}$ within $15 \%$. In this scenario, the total baryon asymmetry in the universe would be at a level close to the observed value after including the dark-matter contribution. Please also note that a low-mass hexaquark is hard to distinguish kinematically from a neutron.

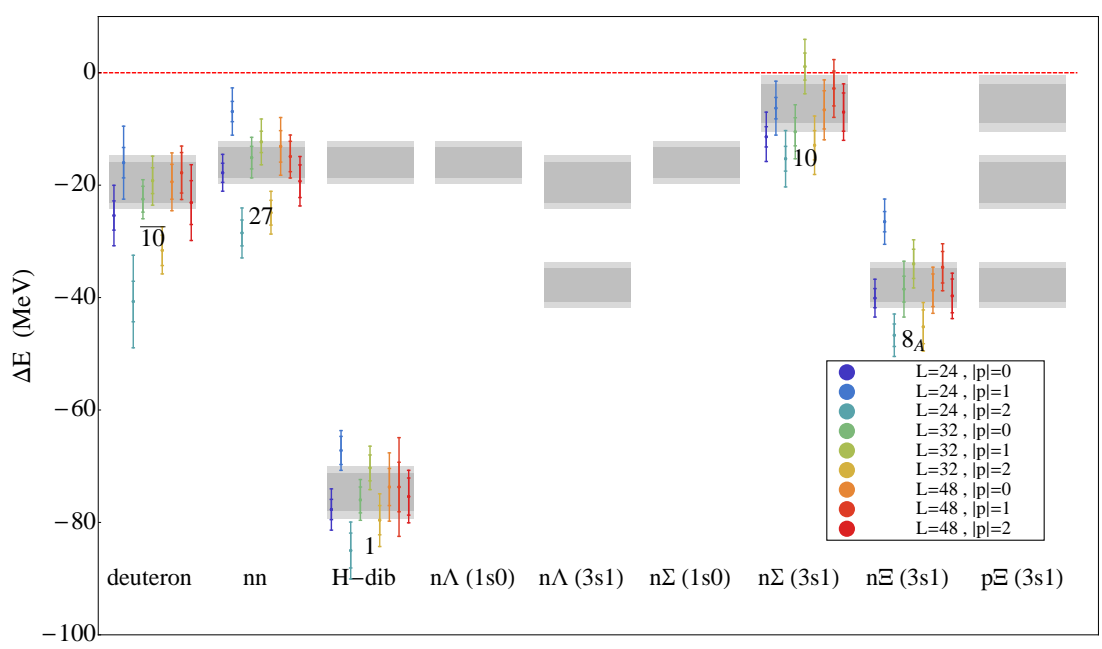

Figure 2: Binding energies of two-baryon systems calculated in lattice QCD. The grey-shaded regions denote statistical uncertainties while the colored error bars show statistical and systematic errors combined. The different colors show results for three lattice dimensions and different momenta [10].

\section{Search Strategy}

The BABAR experiment has searched for the hexaquark $S$ in the process $\Upsilon(2 S, 3 S) \rightarrow$ gluons $\rightarrow$ $S \bar{\Lambda} \bar{\Lambda}$ or $\bar{S} \Lambda \Lambda$ performing a blind analysis of $90 \times 10^{6} \Upsilon(2 S)$ decays and $100 \times 10^{6} \Upsilon(3 S)$ decays. The $\Upsilon(4 S)$ data set of $424.2 \mathrm{fb}^{-1}$ is used to evaluate the off-peak background since according to simulations the contribution of $B$ meson decays to the final mass spectrum is negligible. The $\bar{\Lambda} \bar{\Lambda}$ $(\Lambda \Lambda)$ system is fully reconstructed in the $\bar{p} \pi^{+} \bar{p} \pi^{+}\left(p \pi^{-} p \pi^{-}\right)$decays. The $\Lambda \rightarrow p \pi^{-}$decay has a branching fraction of $\mathscr{B}\left(\Lambda \rightarrow p \pi^{-}\right)=0.64$. With the fully reconstructed $\bar{\Lambda}$ s the recoil mass $m_{\text {rec }}^{2}=\left(p_{\Upsilon}-p_{\bar{\Lambda}}-p_{\bar{\Lambda}}\right)^{2}$ is calculated. Figure 3 (left) shows the topology of the process $e^{+} e^{-} \rightarrow$ $\Upsilon(2 S, 3 S) \rightarrow$ gluons $\rightarrow S \bar{\Lambda} \bar{\Lambda}$. The $S$ lies in the recoil of the $\bar{\Lambda} \bar{\Lambda}$ system.

We select events that have four charged tracks. One additional track is allowed that does not come from the interaction region having a distance of closest approach $D O C A>5 \mathrm{~cm}$. This accounts for particles that are produced in secondary interactions with the detector material. We 
apply loose particle identification criteria to select $p$ or $\bar{p}$. We reconstruct $\bar{\Lambda} \bar{\Lambda}$ or $\Lambda \Lambda$ using the $\bar{p} \pi^{+}$ or $p \pi^{-}$decay. For each $\bar{\Lambda}(\Lambda)$ we require a flight distance significance of $\left|\vec{r}_{\Lambda}\right| / \sigma_{r_{\Lambda}}>5$ where $r_{\Lambda}$ is the flight distance vector. The $\bar{\Lambda}(\Lambda)$ have to point back to interaction region, $\vec{r}_{\Lambda} \cdot \vec{p}_{\Lambda} /\left(\left|\vec{r}_{\Lambda}\right| \vec{p}_{\Lambda} \mid\right)>$ 0.9. To extract the signal we examine the extra neutral energy $E_{\text {extra }}$, which is the energy of all neutral clusters in electromagnetic calorimeter except for a cone around the $S$. Figure 3 (right) shows an event display of a simulated signal event. The pink cone shows the region that is not included in the determination of $E_{\text {extra }}$. For signal we require $E_{\text {extra }}<500 \mathrm{MeV}$. We use the sample $E_{\text {extra }}>500 \mathrm{MeV}$ for validation.
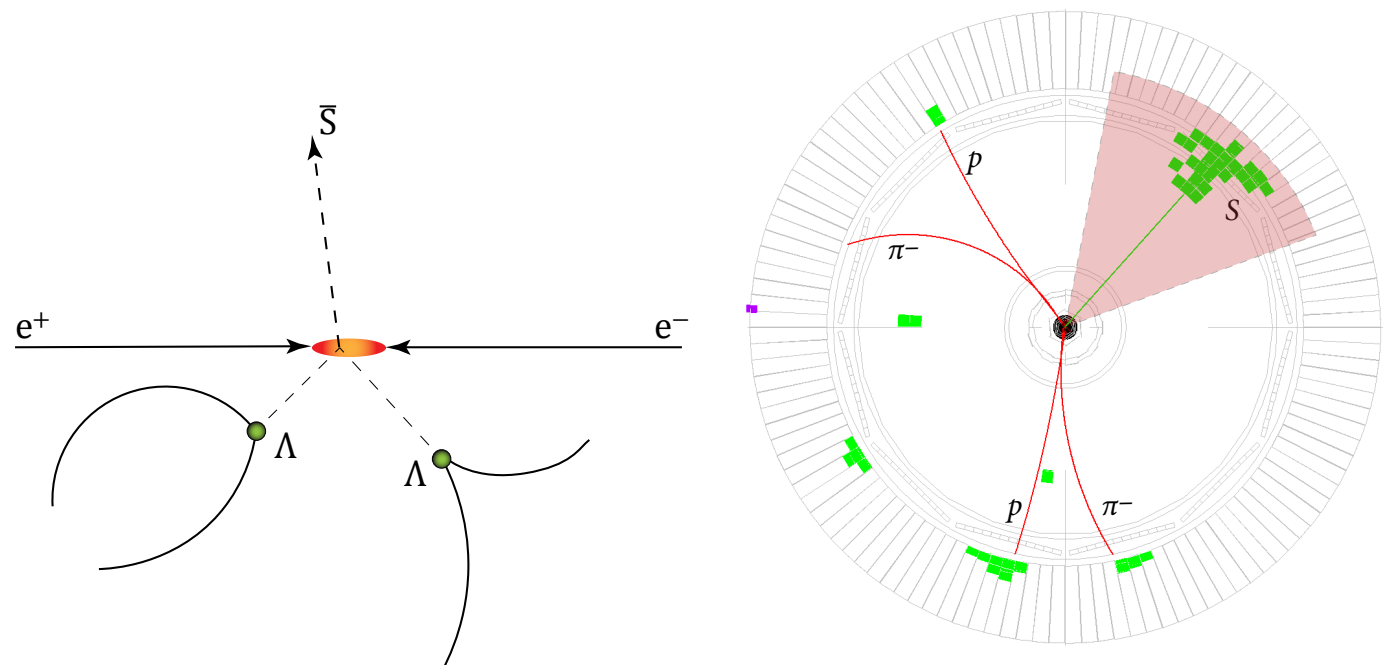

Figure 3: Left: Topology of the process $e^{+} e^{-} \rightarrow \Upsilon(2 S, 3 S) \rightarrow$ gluons $\rightarrow S \bar{\Lambda} \bar{\Lambda}$. Right: Event display of a simulated signal event. Clusters lying in the pink cone are not included in the $E_{\text {extra }}$ calculation.

Figure 4 (left) shows the $E_{\text {extra }}$ distribution after preselection. We see 92 events of which 8 events satisfy $E_{\text {extra }}<500 \mathrm{MeV}$. The $p \pi$ invariant mass shows a true $\Lambda$ signal as depicted in Fig. 4 (right). After performing a kinematic fit of each $\Lambda$ candidate by constraining the mass to the nominal $\Lambda$ mass and requiring a common point in the beam spot leaves four candidates.
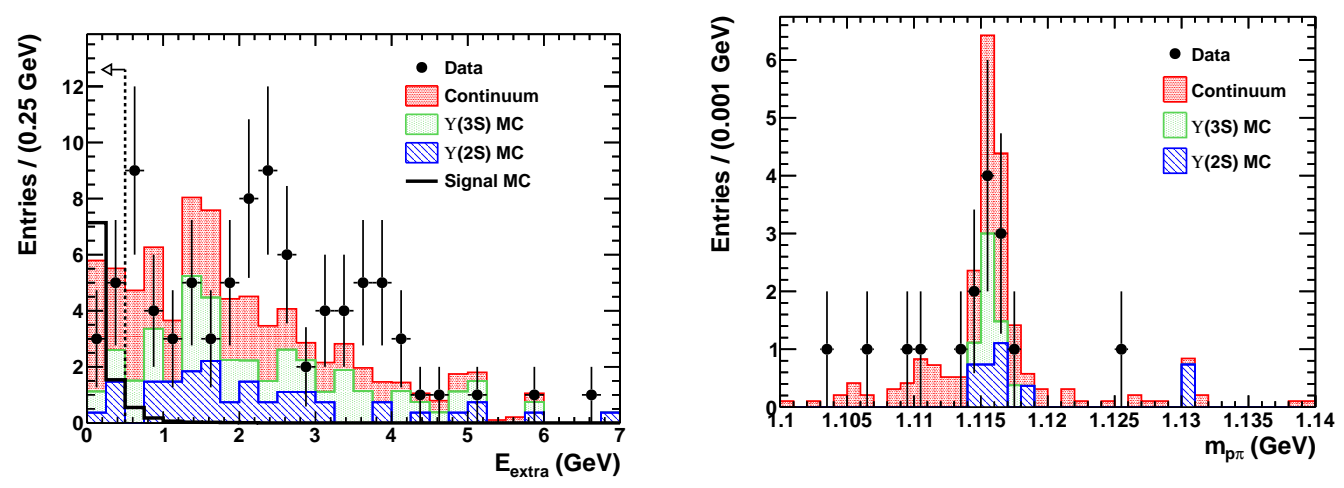

Figure 4: Left: The distribution of extra energy in the calorimeter $E_{\text {extra }}$ after preselection. Right: The $p \pi$ invariant mass of all candidates that satisfy $E_{\text {extra }}<500 \mathrm{MeV} / \mathrm{c}^{2}$. 
Table 1: Systematic uncertainties.

\begin{tabular}{|l|c|}
\hline \hline Source & Systematic error \\
\hline$S$ angular distribution modeling & $5-8 \%$ \\
$S$ particle type modeling & $8-11 \%$ \\
$\Lambda$ reconstruction & $4 \%$ per $\Lambda$ \\
Monte Carlo statistics & $2 \%$ \\
$\Lambda$ branching fraction & $1.6 \%$ \\
proton particle identification & $1 \%$ per $p$ \\
Number of $\Upsilon(2 S, 3 S)$ & $0.6 \%$ \\
\hline \hline
\end{tabular}

\section{Efficiency and Backgrounds}

For simulation of the signal we use dedicated signal Monte Carlo samples that were generated both with the decay amplitude provided by Farrar [11] and with phase space. We model the hexaquark $S$ as a neutron or as a neutrino that has no interaction with the detector material. The efficiency including $\mathscr{B}\left(\lambda \rightarrow p \pi^{-}\right)^{2}$ varies from $7.2 \%$ at threshold to $8.2 \%$ near $2 \mathrm{GeV} / \mathrm{c}^{2}$ and is mainly driven by the geometric acceptance. The resolution of the recoil mass is $100 \mathrm{MeV} / \mathrm{c}^{2}$. The background originates from $q \bar{q}$ continuum with $q=u, d, s, c$ or from $\Upsilon(2 S)$ and $\Upsilon(3 S)$ decays. We use $\Upsilon(4 S)$ decays for $q \bar{q}$ continuum determination since the signal contribution is negligible. We scale the $\Upsilon(2 S)$ and $\Upsilon(3 S)$ Monte Carlo samples so that the Monte Carlo and $q \bar{q}$ continuum matches the $E_{\text {extra }}$ sideband. A residual background consists of $\bar{\Lambda} \bar{\Lambda}(\Lambda \Lambda) X$ events in which the $\bar{\Lambda}(\Lambda)$ decays to $\bar{n}(n) \pi^{0}$. This background may pass or fail the $E_{\text {extra }}$ selection.

\section{Systematic Uncertainties}

Table 1 summarizes the different types of systematic uncertainties. The largest effects come from modeling the hexaquark production amplitude and the angular distribution. The total systematic error ranges from $12.8 \%$ to $16.1 \%$.

\section{Validation and Results}

Figure 5 (left) shows the distribution of the recoil mass $m_{\text {rec }}$ squared for the validation sample for which $E_{\text {extra }}>500 \mathrm{MeV} / \mathrm{c}^{2}$. In the $E_{\text {extra }}$ sideband we observe zero background events in the $m_{\text {rec }}$ signal region. Figure 5 (right) shows $m_{r e c}$ squared for $E_{\text {extra }}<500 \mathrm{MeV} / \mathrm{c}^{2}$ in which we see no events in the signal region. The four candidates populate the high mass region. To illustrate how a signal would look like, we performed a simulation of $S$ with a mass of $1.6 \mathrm{GeV} / \mathrm{c}^{2}$ and a branching fraction of $\mathscr{B}(\Upsilon(n S) \rightarrow S \bar{\Lambda} \bar{\Lambda})=10^{-7}$. We see no evidence for hexaquarks in the decays $\Upsilon(2 S, 3 S) \rightarrow S \bar{\Lambda} \bar{\Lambda}$. We use the profile likelihood method to set branching fraction upper limits at $90 \%$ confidence level in the mass range $0.0-2.05 \mathrm{GeV}$ including systematic errors. Figure 6 shows the branching fraction upper limits at $90 \%$ confidence level as a function of the $S$ mass. The upper limit on the branching fraction at $90 \%$ confidence level is $\mathscr{B}(\Upsilon \rightarrow S \bar{\Lambda} \bar{\Lambda})<1.4 \times 10^{-7}$ at low masses falling off to $\mathscr{B}(\Upsilon \rightarrow S \bar{\Lambda} \bar{\Lambda})<1.2 \times 10^{-7}$ at high masses. 

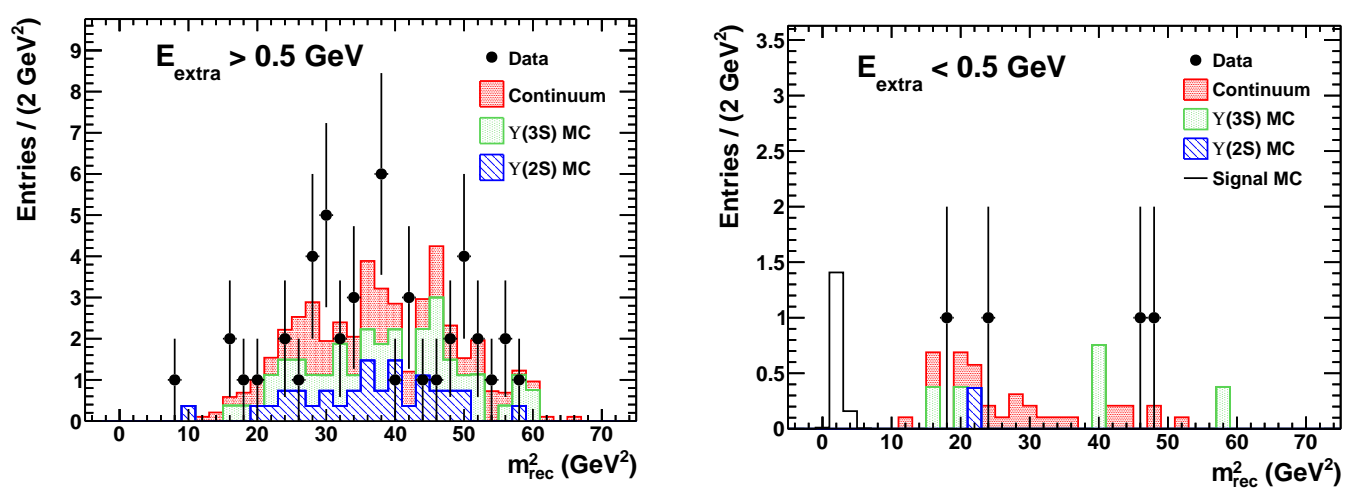

Figure 5: Left: Distribution of the recoil mass for all candidate with $E_{\text {extra }}>500 \mathrm{MeV} / \mathrm{c}^{2}$. Right: Distribution of the recoil mass for signal candidate with $E_{\text {extra }}<500 \mathrm{MeV} / \mathrm{c}^{2}$.

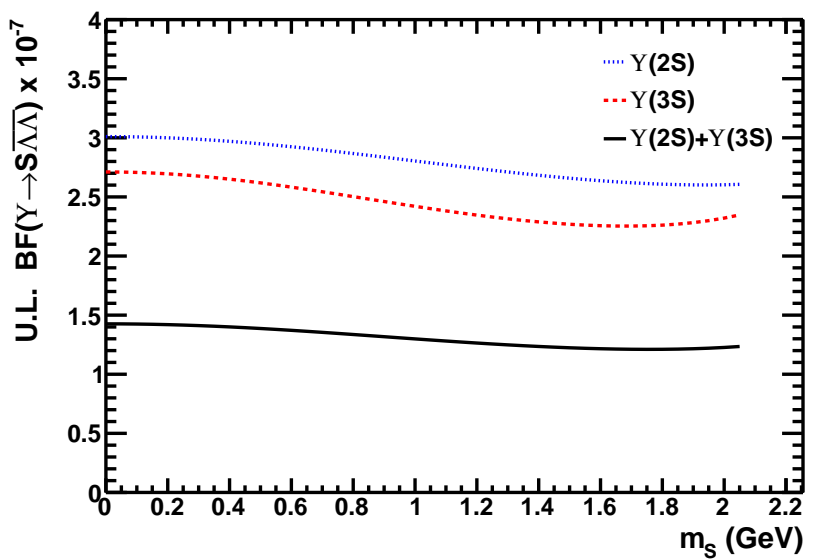

Figure 6: Branching fraction upper limits at $90 \%$ confidence level as a function of $m_{S}$ for $\Upsilon(2 S) \rightarrow S \bar{\Lambda} \bar{\Lambda}$ (blue dotted curve), for $\Upsilon(3 S) \rightarrow S \bar{\Lambda} \bar{\Lambda}$ (red dashed curve) and for both samples combined (black solid line).

\section{Conclusion}

A tightly bound $S=|u u d d s\rangle$ state may be more stable than previously predicted. If $m_{S}<$ $m_{\Lambda}+m_{p}+m_{e}=2.0545 \mathrm{GeV} / \mathrm{c}^{2}$, it may be stable even on cosmological time scales. If it exists, it is a good candidate for dark matter. It is not excluded by searches for the $H$-dibaryon. BABAR has searched for the hexaquark $S$ in $\Upsilon(2 S)$ and $\Upsilon(3 S)$ decays in the recoil against $\bar{\Lambda} \bar{\Lambda}$ using a data sample of 200 million $\Upsilon(2 S)$ and $\Upsilon(3 S)$ events. No evidence is found in $\Upsilon(n S)$ decays for a stable hexaquark state. We see no background events in the signal region and set a stringent branching fraction upper limit of $\mathscr{B}(\Upsilon \rightarrow S \bar{\Lambda} \bar{\Lambda})<(1.2-1.4) \times 10^{-7}$ at $90 \%$ confidence level for $m_{S}<2.05 \mathrm{GeV}$.

\section{References}

[1] M. Gell-Mann, Phys. Rev. 125, 1067 91962).

[2] Belle Collaboration (S.K. Choi et al.), Phys. Rev. Lett. 91, 262001 (2003). 
[3] BES III CollaborationM. Ablikim et al.), Phys. Rev. Lett. 110, 252001 (2013).

[4] Belle Collaboration (S.K. Choi et al.), Phys. Rev. Lett. 100, 142001 (2008); Belle Collaboration (K. Chilikin et al.), Phys. Rev. D 88, 074026 (2013).

[5] LHCb Collaboration (R. Aaij et al.), Phys. Rev. Lett. 122, 222001 (2019).

[6] BABAR Collaboration (J. P. Lees et al.), Phys. Rev. Lett. 122, 072002 (2019).

[7] R. L. Jaffe, Phys. Rev. Lett. 38, 195 (1977).

[8] R. L. Jaffe, Nucl. Phys. A625, 167 (1997).

[9] G.R. Farrar, arXiv:1708.08951 [hep-ph] (2017).

[10] Beane et al., Phys. Rev. D87, 034506 (2013).

[11] G. Farrar, (private communication (2018). 